\title{
Effect of High-Intensity Exercise on Early \\ Responses of Iron and Iron Regulatory Proteins in Young Female Basketball Players
}

\section{Justyna Cichoń}

Poznań University of Physical Education

Joanna Ostapiuk-Karolczuk

Poznań University of Physical Education

\section{Mirosława Cieślicka}

Collegium Medicum in Bydgoszcz, Nicolaus Copernicus University in Toruń

\section{Hanna Dziewiecka}

Poznań University of Physical Education

\section{Anita Marcinkiewicz}

Poznań University of Physical Education

\section{Małgorzata Tafil-Klawe}

Collegium Medicum in Bydgoszcz, Nicolaus Copernicus University in Toruń

\section{Piotr Basta}

Poznań University of Physical Education

\section{Dariusz Maciejewski}

Poznań University of Physical Education

Anna Skarpańska-Stejnborn ( $\square$ ankass@poczta.onet.pl )

Poznań University of Physical Education

\section{Research Article}

Keywords: hepcidin, IL-6, athletes, recovery

Posted Date: March 3rd, 2022

DOI: https://doi.org/10.21203/rs.3.rs-1363213/v1

License: (c) (1) This work is licensed under a Creative Commons Attribution 4.0 International License. Read Full License 


\section{Abstract \\ Background}

Accumulation of physiological stress and the presence of inflammation disturb iron management in athletes during intense training. However, little is known about the mechanisms regulating iron levels in athletes during training periods with low training loads. In the current study, we analyzed the effect of exercise on early responses of iron and iron regulatory proteins during such training periods.

\section{Methods}

The study was performed at the end of competitive phase of training. Twenty-seven trained female basketball players (age, 16,55 $\pm 0,96$ years; weight, $66,40 \pm 13,68 \mathrm{~kg}$ ) participated in the study. The participants performed a maximum exercise test on a treadmill. Blood samples were taken before the test, immediately after exercise, and after $3 \mathrm{~h}$ of restitution. Parameters, such as interleukin (IL) 6 , hepcidin, ferritin, transferrin, hemopexin, and lactoferrin levels, and TIBC and UIBC were determined by using appropriate biochemical tests.

\section{Results}

Intense physical effort performed at the end of the competitive phase led to an increase in iron levels after exercise. The iron levels decreased within $3 \mathrm{~h}$ of exercise. No significant changes in the levels of hepcidin, IL-6, and other proteins related to the regulation of iron levels were observed. Except for iron levels, only TIBC levels significantly increased after exercise and decreased to baseline level during rest period.

\section{Conclusions}

The results suggest that at the end of the competitive phase of training, when training loads are much lower than those at the beginning or in the middle of competitive phase, the post-exercise increase in iron levels is not necessarily accompanied by a simultaneous increase in the levels of hepcidin or inflammatory markers. Ferritin may be the source of iron ions and transferrin may be responsible for their return to baseline. However, both the source of the released iron ions and the mechanism responsible for their regulation require further research.

\section{Background}

Athletes experience disturbances of systemic iron homeostasis, especially during training periods characterized by intense training loads. This phenomenon has been called "sports anemia". Its causes and underpinning biochemical mechanisms are poorly understood. Based on the available data, the risk 
of depletion of iron stores is especially high in women and adolescents, which may lead to severe iron deficiency. Insufficient reserves of iron in the body can reduce athletic performance, which may be manifested as fatigue, exercise intolerance, or even cognitive function impairment (Murray-Kolb, Beard, 2007; Ausperger et al., 2013).

Several mechanisms may be responsible for iron loss during exercise, including hemolysis, hematuria, sweating, and gastrointestinal bleeding (Yusof, 2007). Oxidative stress induced by exercise and the associated inflammation result in biochemical changes in the erythrocyte cell membrane, leading to its increased susceptibility to damage and increased intravascular hemolysis (Van Iperen et al., 2001, Lippi, Sanchis-Gomar, 2019). According to Barros et al. (2012), hemolysis is not the only source of the increased concentration of iron ions in serum during intense exercise. They showed that in individuals with elevated after exercise iron concentrations, only approximately $20 \%$ of iron ions in serum come from hemolysis. The authors suggested that ferritin and transferrin are the main source of iron ions in this case. Further, Reif (1992) demonstrated that free oxygen radicals (reactive oxygen species, ROS) are primarily responsible for the release of iron ions from ferritin.

Transferrin and ferritin bind nearly all iron circulating in the serum. Under physiological conditions, this chelation makes the iron soluble, prevents iron-mediated free-radical toxicity, and facilitates the transport of iron into cells. Hence, the total iron-binding capacity (TIBC) of serum reflects serum transferrin levels (Soldin et al., 2004, Ponka, 1997). Another factor involved in iron homeostasis is the labile iron pool (LIP). LIP is a pool of weakly chelated, low-molecular weight iron that is redox-active. Alterations in the LIP size may lead to a variety of injurious processes, such as oxidative stress (Kruszewski et al., 2003, Kakhlon et al., 2002).

Post-exercise inflammatory response is mainly manifested through elevated levels of interleukin (IL) 6, a cytokine with a major role in the regulation of iron management (Nemeth et al., 2004). IL-6 is a key regulator of the activity of the iron-regulating hormone hepcidin. This hormone adversely affects the activity of the main iron export protein, ferroportin, which leads to a decrease of iron absorption in the intestine, and inhibition of iron release from hemolyzed red blood cells and internal organs that store iron (Nemeth et al., 2004b).

Hemopexin and lactoferrin are also involved in the regulation of iron levels. They capture free heme or iron ions, and thus contribute to the regulation of inflammation (Lin et al. 2012; Rosa et al., 2017). Both proteins reduce macrophage production of pro-inflammatory cytokines, including IL-6. They thus affect the activity of ferroportin - positively; and ferritin - negatively, preventing intracellular iron overload and inhibiting the development of inflammation (Rosa et al., 2017).

Considering the potential toxicity of systemic iron overload, as well as the negative impact of iron deficiency on the physical performance of athletes, the regulatory role of hepcidin in iron management and its relationship with inflammation are of considerable interest (Pal et al., 2020; Larsuphrom et al., 2021). However, proper iron management also depends on many other factors, including the levels of proteins related to iron uptake and transport. 
To date, studies into the changes in iron levels or the levels of proteins responsible for the regulation of iron homeostasis have primarily focused on pre- and post-exercise values and, predominantly, on the restitution period $24 \mathrm{~h}$ post-exercise (Zügel et al., 2020; Skarpańska-Stejnborn et al., 2015; Auersperger et al, 2013). Only few studies have focused on the early restitution period. Such studies mainly consider the relationship between the iron status and the levels of hepcidin (Peeling et al., 2009; Badenhorst et al., 2015). However, as a caveat to interpreting the results of such studies, the reported data hold for athletes during periods of high-stress training, and the observed levels of iron metabolism markers were not compared with those in completely rested athletes.

Further, based on the available data, the iron indices and hematological parameters in elite athletes change during the training cycle, depending on the training intensity and frequency (Banfi et al., 2005, 2006; Candau et al., 1992). Malcovati et al. (2003) assessed the effect of exercise and training phase on hematological parameters and iron homeostasis indicators in 923 professional football players. They found that in well-trained athletes, most variables [e.g., ferritin, hemoglobin, and mean corpuscular volume (MCV)] were highest at the start of the playing season and then decreased. It seems that the direction of changes in hematological parameters and indicators of serum iron homeostasis after exercise is determined by the training loads used in individual training cycles. However, as mentioned above, most of the studies conducted to date have focused on the competition period, where an increase in inflammation is observed, which may greatly affect post-exercise iron management disorders (Zügel et al., 2020; Skarpańska-Stejnborn et al., 2015). Little is known about whether these changes are also sustained at the end of the competition period, when the training loads are greatly reduced.

We hypothesized that the changes in the level of iron and its regulatory proteins may depend not only on the acuity of exercise but also with respect to the training load. Accordingly, the current study aimed to assess the impact of intense effort performed at the end of the competition period on iron levels and selected parameters related to the regulation of serum iron homeostasis in trained basketball players.

\section{Methods}

\section{Participants and study design}

Twenty-seven female elite basketball players participated in the study. All participants belong to the youth groups of ENEA AZS AJP Gorzów Wielkopolski (1st League and Premier League) (Table 1). The research was conducted at the end of the competition period. All players were on the same team and were subjected to the same training regime. Specifically, the training sessions took place every day, in the afternoon, and lasted from 1.5 to $2 \mathrm{~h}$. 
Table 1

Basic characteristics of study participants $(\mathrm{N}=27)$

\begin{tabular}{|ll|}
\hline Parameter & $\overline{\mathbf{x}} \pm$ SD \\
\hline Age (years) & $16.55 \pm 0.96$ \\
\hline Body mass $(\mathrm{kg})$ & $66.40 \pm 13.68$ \\
\hline Height $(\mathrm{cm})$ & $173.45 \pm 5.14$ \\
\hline Time $(\mathrm{s})$ & $634.33 \pm 96.18$ \\
\hline $\mathrm{HR}$ & $192.13 \pm 12.29$ \\
\hline $\mathrm{V}_{\max }(\mathrm{Bpm})$ & $12.85 \pm 1.14$ \\
\hline $\begin{array}{l}\text { Note. } \text { Values are presented as the mean } \pm \mathrm{SD} ; \text { Time, time to peak intensity exercise; } \mathrm{HR}_{\text {max }} \text {, maximal } \\
\text { heart rate; } \mathrm{V}_{\text {max }} \text { maximal run velocity }\end{array}$ \\
\hline
\end{tabular}

\section{Physical performance test}

All participants performed a maximum stress test on HP Cosmos Treadmill. During the test, the aerobic capacity of participants was assessed. The test protocol was as follows: the starting speed of the treadmill for the runners was $8.0 \mathrm{~km} / \mathrm{h}$; it was then increased every 2 min by $1.0 \mathrm{~km} / \mathrm{h}$, until exhaustion. The participants were verbally encouraged to continue for as long as possible. Heart rate (bpm) was recorded using a sport tester (Polar PE 3000) (Table 1).

\section{Blood analysis}

Blood samples were collected from study participants from the antecubital vein before exercise (Preexercise), in the first minute after exercise (Post-exercise), and after $3 \mathrm{~h}$ of restitution ( $3 \mathrm{~h}$ Recovery). Hematological variables, such as red blood cell count (RBC), hemoglobin ( $\mathrm{Hb})$, hematocrit (HCT), mean corpuscular hemoglobin $(\mathrm{MCH})$, mean corpuscular hemoglobin concentration (MCHC), mean corpuscular volume (MCV), red cell distribution width (RDW), mean platelet volume (MPV), and platelet count (PLT), were analyzed using the MYTHIC 18 Haematology Analyser (Orphee Medical, Geneva, Switzerland). Iron concentration and TIBC (Total Iron Binding Capacity) were determined using colorimetric method with chromogens (cat. no. 1-418-01-50 and 1-421-0060, respectively; BioMaxima, Lublin, Poland).

Unsaturated iron-binding capacity (UIBC) was calculated using the formula: UIBC $=$ TIBC - Fe. Transferrin saturation was calculated as serum iron/TIBC. Enzyme-linked immunosorbent assay kits and Thermo Scientific Multiscan GO microplate spectrophotometer (Fisher Scientific, Vantaa, Finland) were used to determine serum levels of the following molecules: IL-6, transferrin, hemopexin, and lactate dehydrogenase (LDH) (SunRed Biotechnology Co., Ltd (Shanghai, China); hepcidin, myoglobin, and ferritin (DRG International Inc., CITY, STATE, USA); and lactoferrin (AssyPro, St Charles, MO, USA).

\section{Statistical analysis}


Statistical analyses were performed using STATISTICA v. 13.0 software package (Stat-Soft, Kraków, Poland). Descriptive statistics, including mean and SD, were used to visualize immediate trends and patterns. Shapiro-Wilk test was used to examine the normal distribution of variables. Levene's test was used to verify the homogeneity of variance. If significant changes were detected by one-way analysis of variance (ANOVA), Fisher's post hoc test was used to identify the source of significant differences. Nonparametric Friedman ANOVA was used to analyze variables with non-normal distribution. Pearson's coefficient of linear correlation was calculated for correlation analysis. For all tests, the statistical significance threshold was set at $p \leq 0.05$.

\section{Results}

Iron levels significantly increased after exercise $(p<0.05)$; in the following $3 \mathrm{~h}$ of restitution, the iron levels decreased and reached values close to the baseline. No significant changes in the levels of hepcidin and IL-6 were observed during the study (Fig. 1).

No significant changes in transferrin levels were observed; however, TIBC slightly increased after exercise and then significantly decreased in the $3 \mathrm{~h}$ after exercise $(p<0.05)$. Transferrin saturation significantly increased immediately after exercise $(p<0.05)$ (Fig. 2).

A gradual and significant decrease in $\mathrm{Hb}$ levels was observed $(p<0.05)$, accompanied by a constant and significant increase in the concentration of myoglobin $(p<0.001$, Pre-exercise vs. $3 \mathrm{~h}$ Recovery; $p<0.001$ Post-exercise vs. 3 h Recovery) (Fig. 3).

For other parameters involved in iron metabolism, a significant decrease in the levels of lactoferrin was observed at the two time points after exercise compared to the baseline values $(p<0.05)$. The levels of LDH increased after exercise $(p<0.05)$ and then significantly decreased $(p<0.05)$ compared to the baseline values. No significant changes in ferritin concentration, transferrin, and UIBC saturation values were observed (Table 2). 
Table 2

Changes in iron metabolism status during acute exercise

\begin{tabular}{|lccc|}
\hline Parameter & Preexercise & Post-exercise & 3 h Recovery \\
\hline Lactoferrin $[\mathrm{ng} / \mathrm{mL}]$ & $136.46 \pm 20.26$ & $86.54 \pm 6.73^{\mathrm{a}}$ & $86.20 \pm 5.51^{\mathrm{c}}$ \\
\hline Hemopexin $[\mathrm{ng} / \mathrm{mL}]$ & $902.88 \pm 642.85$ & $906.31 \pm 706.31$ & $918.07 \pm 752.40$ \\
\hline $\mathrm{LDH}[\mathrm{U} / \mathrm{L}]$ & $266.47 \pm 32.28$ & $280.26 \pm 46.01^{\mathrm{a}}$ & $264.49 \pm 29.75^{\mathrm{c}}$ \\
\hline Ferritin $[\mathrm{ng} / \mathrm{mL}]$ & $90.78 \pm 13.99$ & $91.68 \pm 15.20$ & $89.54 \pm 14.89$ \\
UIBC & $237.45 \pm 75.62$ & $239.33 \pm 98.18$ & $218.13 \pm 74.87$ \\
\hline $\begin{array}{l}\text { Note. } \text { Values are presented as the mean } \pm \mathrm{SD} \text {; } \\
\text { Recovery; }{ }^{\mathrm{c}} \text { Post-exercise vs. Recovery }\end{array}$ & & \\
\hline
\end{tabular}

Among the blood parameters examined, a significant increase in RBC after exercise was observed compared to the resting values $(p<0.05)$. During the restoration period, the RBC dropped significantly below the values observed at rest $(p<0.001)$. Similarly, significant changes were observed for HCT $(p<$ 0.05 , Pre-exercise vs. Post-exercise; $p<0.001$, Pre-exercise vs. 3 h Recovery; $p<0,001$, Post-exerciseRecovery); MCV ( $p<0.05$, Pre-exercise vs. Post-exercise; $p<0.001$, Pre-exercise vs. 3 h Recovery; $p<0,001$, Post-exercise-Recovery); and MPV ( $p<0.05$, Post-exercise vs. $3 \mathrm{~h}$ Recovery; $p<0.001$, Pre-exercise vs. $3 \mathrm{~h}$ Recovery) (Table 3).

Table 3

Changes in RBC parameters during acute exercise

\begin{tabular}{|llll|}
\hline Parameter & Preexercise & Post-exercise & 3 h Recovery \\
\hline RBC & $4.27 \pm 0.316$ & $4.40 \pm 0.328^{\mathrm{a}}$ & $3.97 \pm 0.384^{\mathrm{b}, \mathrm{c}}$ \\
\hline $\mathrm{HCT}$ & $37.26 \pm 2.509$ & $38.21 \pm 2.432^{\mathrm{a}}$ & $34.17 \pm 3.012^{\mathrm{b}, \mathrm{c}}$ \\
\hline $\mathrm{MCV}$ & $87.49 \pm 3.919$ & $86.96 \pm 3.95^{\mathrm{a}}$ & $86.23 \pm 3.817^{\mathrm{b}, \mathrm{c}}$ \\
\hline $\mathrm{MCH}$ & $31.32 \pm 1.55$ & $31.16 \pm 1.721$ & $31.09 \pm 1.784$ \\
\hline $\mathrm{MCHC}$ & $35.80 \pm 0.483$ & $35.82 \pm 0.661$ & $36.06 \pm 1.139$ \\
\hline RDW & $13.06 \pm 0.72$ & $13.36 \pm 0.715$ & $13.23 \pm 0.744$ \\
\hline MPV & $8.048 \pm 0.539$ & $7.99 \pm 0.546$ & $7.79 \pm 0.571^{\mathrm{c}}$ \\
\hline $\begin{array}{l}\text { Note. Values are presented the mean } \pm \text { SD; } \\
\text { c Post-exercise vs. Recovery }\end{array}$ & & \\
\hline
\end{tabular}

Finally, TIBC was positively correlated with iron levels $(r=0.2826 ; p=0.014)$, and negatively correlated with hepcidin levels $(r=-0.2322 ; p=0.045)$ (Fig. 4). 


\section{Discussion}

We here aimed to assess the impact of intense exercise performed at the end of the competition period on iron and iron regulatory proteins level. We found that intense exercise caused a short-term increase in iron ion levels, with a simultaneous increase in transferrin saturation and a slight increase in TIBC.

In recent years, many studies have been conducted to determine the potential impact of intense exercise on hepcidin levels (Nemeth et al., 2004a, Nemeth et al., 2004b, Ausperger et al., 2013). The obtained data indicate that hepcidin levels are associated with an observable increase in iron levels, and reach a maximum value 3-6 h after exercise. Hepcidin levels are also associated with a post-exercise increase in IL-6 levels (Larsuphrom et al., 2021). According to Nemeth et al. (2004), IL-6 levels correspond to the release of hepcidin during exercise-induced inflammation. However, this phenomenon was not confirmed in the current study, as we observed a significant post-exercise increase in iron levels, with a simultaneous lack of hepcidin and IL- 6 responses. The lack of a significant increase in IL- 6 levels after the exercise test was surprising but not implausible. In one study (Peak, et al., 2015), the concentration of proinflammatory cytokines, including IL-6, in the serum significantly increased after a long-term endurance effort, e.g., a marathon or triathlon. This response may be significantly less pronounced after a short vigorous exercise (Kim et al., 2015). In addition, the training frequency of elite athletes is often so high that the short time between consecutive sessions may not be sufficient to achieve full recovery; this, in turn, may lead to the accumulation of harmful metabolites and an increase in the inflammatory response (Dupuy et al., 2018).

It is worth emphasizing that the current study involved highly trained women the final stage of the starting period, when the exercise load was reduced. In addition, the exercise test performed by the players on a treadmill was characterized by high intensity, but a relatively short duration. The lack of significant changes in IL-6 levels may be responsible for the lack of hepcidin level increase observed in other studies. It is also important to note that trained athletes show a specific adaptation to exercise, i.e., increased resting levels of hepcidin. Sandström et al. (2017) compared groups of training and nontraining women, and showed that the resting hepcidin levels in the training women were approximately $40 \%$ higher than those in non-training women, indicating that the higher values in training individuals are a result of training adaptation. Similar to our findings, no significant changes in IL- 6 and hepcidin levels were observed by Troadec et al. (2009) after a 45-min submaximal ergometer test of healthy volunteers. In turn, Kasprowicz et al. (2013) reported a significant increase in IL-6 levels in athletes after a $100 \mathrm{~km}$ run (ultra-marathon), which was not accompanied by an increase in hepcidin levels. A major involvement of hepcidin in the regulation of iron homeostasis was also demonstrated by Peeling et al. (2014), who showed that changes in hepcidin levels post-exercise depend not only on the duration of exercise or IL-6 levels, but also on the initial serum iron and ferritin levels. In athletes with lower baseline iron and ferritin levels, the increase in hepcidin was significantly lower than that in athletes with higher baseline iron and ferritin levels. Whereas, the results of the current study suggest that the observed significant increase in post-exercise iron levels is not a strong enough stimulus to activate hepcidin dependent mechanism of iron regulation in well-rested athletes. 
The disturbance of iron metabolism, resulting from the presence of inflammation manifested by increased IL- 6 levels, is, as mentioned above, associated with training periods characterized by high loads (preparation and competition period). In the current study, all measurements were conducted at the final stage of the starting period. This stage of training is characterized, as already mentioned, by a reduced training load and, therefore, may impact the biochemical and morphological parameters of the subjects.

Ostojic (2009) showed that in highly trained athletes (footballers) during the preconditioning period, most of the parameters related to iron management are higher than those in the playing season associated with higher loads and, thus, fatigue. Importantly, Banfi et al. (2006) observed seasonal variability of iron metabolism parameters related to different training loads. It can be assumed that the management of iron ions during the restitution period may be regulated by different mechanisms than those operational during intense training or competition period.

We did not note any changes in ferritin levels in the current study. Changes in ferritin levels, especially their reduction, are frequently observed among athletes and indicate iron store depletion (Sim et al., 2019). Ferritin levels positively correlate with tissue iron stores, including the bone marrow, and hence, they are a good indicator of iron reserves in the body (Magazanik et al., 1998). Since muscle oxygen consumption increases during intense exercise, which increases ROS levels, it is likely that ROS may contribute to the release of some of the iron stored in ferritin (Borkowska et al., 2011).

An interesting observation of the current study was the lack of changes in transferrin levels during the analyzed period, with a simultaneous change in the parameters directly related to its activity, i.e., TIBC and transferrin saturation after exercise. Transferrin saturation significantly increased immediately after exercise, while changes in TIBC (reflecting changes in iron levels) increased immediately after exercise and then significantly decreased after a $3 \mathrm{~h}$ rest period. In addition, we observed a significant positive correlation between TIBC and iron levels, and a negative correlation between TIBC and hepcidin. Typically, $60 \%$ of the serum transferrin pool is in the form of apotransferrin, i.e., a protein that is not saturated with iron. Apotransferrin is responsible for capturing free iron ions, including those from damaged hemoglobin (Huebers et al., 1983). The increase in transferrin saturation and changes in TIBC levels observed in the current study in parallel with the increased iron levels may indicate that iron capture by apotransferrin is the main mechanism responsible for the reduction of serum iron levels to the baseline values during the restitution period. The decrease in TIBC observed after a $3 \mathrm{~h}$ rest suggests that the iron levels have returned to the baseline values.

According to Hymes et al. (1986), a TIBC increase in athletes suggests an increased need for iron. The TIBC values increase with decreasing iron stores indicatingthat the iron is being moved to the stored iron pool.

Considering that the observed changes in iron ion level occurred within $3 \mathrm{~h}$ of exercise, it can be concluded that capturing iron by apotransferrin is a relatively rapid mechanism of iron level regulation and may be related to the high training level of the participants. The iron released during exercise was entirely captured by the circulating transferrin in the serum, without the need to activate additional 
regulatory mechanisms, e.g., increasing the hepcidin levels. Changes in transferrin saturation or TIBC are often used to characterize the dynamic changes in iron levels during training, or to observe the effects of a single, intense exercise (Deruisseau et al., 2004; Haymes et al., 1986). However, the reports on TIBC and transferrin saturation in athletes are inconsistent. Whereas we have observed changes in TIBC similar to those reported here in a previous study involving a group of rowers (Skarpańska et al., 2015), others have observed the involvement of the IL-6-hepcidin axis. Deruisseu et al. (2004) reported no changes in iron levels or parameters characterizing its economy, such as transferrin, TIBC, or transferrin saturation, in participants after 3 weeks of strength training. On the other hand, Podgórski et al. (2015) demonstrated a simultaneous increase in TIBC and iron levels throughout the annual training cycle of field hockey players.

Since iron deficiency, often observed in athletes, may negatively impact not only the oxygen transport and immune defenses, but also the individual's sports performance, monitoring these parameters during training is important (Peeling et al., 2008).

Lactoferrin is another protein that regulates the concentration of iron ions in the body. Its levels rapidly increase in response to inflammation (Drago-Serrano et al., 2017). The data presented herein indicated lack of inflammation in the study participants in the first few hours after exercise, which would explain the lack of the expected increase in lactoferrin levels after exercise.

The iron balance is also characterized by red cell parameters, such as $\mathrm{RBC}, \mathrm{HTC}, \mathrm{Hb}$, and myoglobin. In a study of Boyadjiev and Tarałow (2000), the values of hematological variables in trained women were lower than those in the untrained control group. In addition, they observed differences in these values among athletes from different disciplines, with the lowest values noted for representatives of highintensity training disciplines, including rowers and swimmers. The intense physical effort that the basketball players were subjected to in the current study resulted in a significant but short-term increase in $\mathrm{RBC}, \mathrm{Hb}$, and $\mathrm{HTC}$, and their levels were comparable with the baseline values during the $3 \mathrm{~h}$ rest period.

In sports players representing various disciplines, increasing inflammation disturbs iron management, which is manifested by a reduction in iron levels, and may lower the values of hematological parameters (RBC, $\mathrm{Hb}$, and $\mathrm{HTC}$ ). The factors responsible for the decline in these parameters also include intravascular hemolysis during exercise (Telford et al., 2003). Further, Mairbäurl (2013) showed that increased oxygen demand in the muscle during intense exercise may be compensated by an increased blood flow and repeated hypoxic state, which increases the synthesis of erythropoietin in the kidney, leading to an increase in RBC production and $\mathrm{Hb}$ levels. In the current study, we observed a significant and continuous increase in myoglobin levels in study participants. According to the available literature data, this parameter is an indicator of muscle fiber damage and increases as a result of intense exercise (Nishiie-Yano et al., 2019; Buratti et al., 2015). Furthermore, Eichner (1992) pointed out that hemoglobin levels in athletes, especially endurance athletes, tend to be lower than those in untrained individuals. The hemolysis that occurs during intense exercise may lead to disturbances in both, hematological parameters and iron metabolism, leading to iron deficiency. Hence, iron supplementation is often 
recommended for athletes, especially for women, even in the absence of anemia symptoms (Brownile et al. 2004, Hinton, Sinclair, 2006).

The observed increase in RBC and $\mathrm{Hb}$ values after physical exertion, along with simultaneously increased iron levels, may indicate a response that is not disturbed by inflammation. They may also suggest that the initial iron levels in the group of female players selected for the current study were sufficient and stable.

\section{Conclusions}

Intense exercise performed by highly qualified athletes during training period focused on regeneration caused a short-term increase in iron ion levels, with a simultaneous increase in transferrin saturation and a slight increase in TIBC. Both parameters returned to baseline values during the $3 \mathrm{~h}$ recovery period. However, no changes in the levels of IL-6 and hepcidin, and transferrin and ferritin, were observed. Postexercise increase in hematological parameters suggests that ferritin, and not post-exercise hemolysis, is the source of iron ions. This could indicate that the study participants were not iron-deficient. Hence, the accumulation of physical loads during intense training periods, and not a single physical effort, may determine the directionality of changes in the iron homeostasis parameters. Further research is needed into the ways of addressing the disturbances of iron metabolism in athletes, not only during periods of intense training (characterized by a high level of fatigue), but also during periods of rest and regeneration, as well as into the sequence of reactions responsible for restoring systemic iron homeostasis.

\section{List Of Abbreviations}

\section{RBC}

red blood cell count

$\mathrm{Hb}$

hemoglobin

$\mathrm{HR}_{\text {max }}$

maximal heart rate in beats per minute

HTC

hematocrit

$\mathrm{MCH}$

mean corpuscular hemoglobin

$\mathrm{MCHC}$

mean corpuscular hemoglobin concentration

MCV

mean platelet volume

\section{MPV}

mean platelet volume

PLT 
platelet count

RDW

red cell distribution width

TIBC

Total Iron Binding Capacity

UIBC

Unsaturated iron-binding capacity

bpm

Heart rate (beats per minute)

LDH

lactate dehydrogenase

Vmax

maximal run velocity in kilometers per hour

\section{Declarations}

\section{Ethics approval and consent to participate}

The study was conducted in accordance with the Declaration of Helsinki, and its protocol was approved by the local Ethics Committee at Poznań University of Medical Sciences (decision no. 714/18 of 14 June 2017). All procedures and potential risks were discussed with the participants before the study. Informed consent was obtained from all parents or legal guardians and subjects before participation in the study.

\section{Consent for publication}

Not applicable

\section{Availability of data and materials}

Due to ethical concerns the datasets generated and/or analyzed during the current study supporting data cannot be made openly available however, are available from the corresponding author on reasonable request.

\section{Competing interests}

The authors declare that they have no competing interests

\section{Funding}

The Regional Operational Program - Lubuskie 2020; Priority axis 1 "Economy and Innovation"; Action 1.1. Research and Innovation. https://rpo.lubuskie.pl/-/zakonczenie-oceny-merytorycznej-w-ramach-konkursunr-rplb-01-01-00-iz-00-08-k01-16

\section{Authors' contributions}


ASS conceived and planned the experiment. AM carried out laboratory analysis. JC, JOK contributed to the statistical analysis, and took lead in writing the manuscript with consultation with ASS and MTK, PB and DM collected the data, HD visualization, ASS and MTK project administration, ASS and MC funding acquisition. All authors have read and agreed to the published version of the manuscript.

\section{Acknowledgements}

Not applicable

\section{Authors' information (optional)}

Not applicable

\section{References}

1. Auersperger I, Skof B, Leskosek B, et al.: Exercise-induced changes in iron status and hepcidin response in female runners. PLoS One 2013, 8:e58090.

2. Banfi G, Del Fabbro M, Mauri C, et al.: Haematological parameters in elite rugby players during a competitive season. Clin Lab Haematol 2006, 28:183-8.

3. Banfi G, Dolci A, Freschi M, et al.: Immature reticulocyte fraction (irf) monitored in elite athletes during a whole season. Clin Lab Haematol 2005, 27:213-4.

4. Barros MP, Ganini D, Lorenco-Lima L, et al.: Effects of acute creatine supplementation on iron homeostasis and uric acid-based antioxidant capacity of plasma after wingate test. J Int Soc Sports Nutr 2012, 9:25.

5. Borkowska A, Sielicka-Dudzin A, Herman-Antosiewicz A, et al.: P66shc mediated ferritin degradationa novel mechanism of ros formation. Free Radic Biol Med 2011, 51:658-63.

6. Brownlie Tt, Utermohlen V, Hinton PS, et al.: Tissue iron deficiency without anemia impairs adaptation in endurance capacity after aerobic training in previously untrained women. Am J Clin Nutr 2004, 79:437-43.

7. Buratti P, Gammella E, Rybinska I, et al.: Recent advances in iron metabolism: Relevance for health, exercise, and performance. Med Sci Sports Exerc 2015, 47:1596-604.

8. Candau R, Busso T, and Lacour JR: Effects of training on iron status in cross-country skiers. Eur J Appl Physiol Occup Physiol 1992, 64:497-502.

9. Deruisseau KC, Roberts LM, Kushnick MR, et al.: Iron status of young males and females performing weight-training exercise. Med Sci Sports Exerc 2004, 36:241-8.

10. Drago-Serrano ME, Campos-Rodriguez R, Carrero JC, et al.: Lactoferrin: Balancing ups and downs of inflammation due to microbial infections. Int J Mol Sci 2017, 18.

11. Dupuy O, Douzi W, Theurot $D$, et al.: An evidence-based approach for choosing post-exercise recovery techniques to reduce markers of muscle damage, soreness, fatigue, and inflammation: A systematic review with meta-analysis. Front Physiol 2018, 9:403. 
12. Haymes EM, Puhl JL, and Temples TE: Training for cross-country skiing and iron status. Med Sci Sports Exerc 1986, 18:162-7.

13. Heffernan SM, Horner K, De Vito G, et al.: The role of mineral and trace element supplementation in exercise and athletic performance: A systematic review. Nutrients 2019, 11.

14. Hinton PS and Sinclair LM: Iron supplementation maintains ventilatory threshold and improves energetic efficiency in iron-deficient nonanemic athletes. Eur J Clin Nutr 2007, 61:30-9.

15. Huebers HA, Huebers E, Csiba E, et al.: The significance of transferrin for intestinal iron absorption. Blood 1983, 61:283-90.

16. Kakhlon $\mathrm{O}$ and Cabantchik ZI: The labile iron pool: Characterization, measurement, and participation in cellular processes(1). Free Radic Biol Med 2002, 33:1037-46.

17. Kim HK, Konishi M, Takahashi M, et al.: Effects of acute endurance exercise performed in the morning and evening on inflammatory cytokine and metabolic hormone responses. PLoS One 2015, 10:e0137567.

18. Kruszewski M: Labile iron pool: The main determinant of cellular response to oxidative stress. Mutat Res 2003, 531:81-92.

19. Larsuphrom $P$ and Latunde-Dada GO: Association of serum hepcidin levels with aerobic and resistance exercise: A systematic review. Nutrients 2021, 13.

20. Lin T, Sammy $F$, Yang $H$, et al.: Identification of hemopexin as an anti-inflammatory factor that inhibits synergy of hemoglobin with hmgb1 in sterile and infectious inflammation. J Immunol 2012, 189:2017-22.

21. Lippi G and Sanchis-Gomar F: Epidemiological, biological and clinical update on exercise-induced hemolysis. Ann Transl Med 2019, 7:270.

22. Magazanik A, Weinstein Y, Dlin RA, et al.: Iron deficiency caused by 7 weeks of intensive physical exercise. Eur J Appl Physiol Occup Physiol 1988, 57:198-202.

23. Mairbaurl $\mathrm{H}$ : Red blood cells in sports: Effects of exercise and training on oxygen supply by red blood cells. Front Physiol 2013, 4:332.

24. Malcovati L, Pascutto $C$, and Cazzola M: Hematologic passport for athletes competing in endurance sports: A feasibility study. Haematologica 2003, 88:570-81.

25. Murray-Kolb LE and Beard JL: Iron treatment normalizes cognitive functioning in young women. Am J Clin Nutr 2007, 85:778-87.

26. Nemeth E, Rivera S, Gabayan V, et al.: II-6 mediates hypoferremia of inflammation by inducing the synthesis of the iron regulatory hormone hepcidin. J Clin Invest 2004, 113:1271-6.

27. Nemeth E, Tuttle MS, Powelson J, et al.: Hepcidin regulates cellular iron efflux by binding to ferroportin and inducing its internalization. Science 2004, 306:2090-3.

28. Nishiie-Yano R, Hirayama S, Tamura M, et al.: Hemolysis is responsible for elevation of serum iron concentration after regular exercises in judo athletes. Biol Trace Elem Res 2020, 197:63-69. 
29. Ostojic SM and Ahmetovic Z: Indicators of iron status in elite soccer players during the sports season. Int J Lab Hematol 2009, 31:447-52.

30. Pal S RP, Pawaria S, Das J, Relayach N.: Prevalence of iron deficiency with or without anemia in female athletes - a review. Eur J Mol Clin Med 2020, 7(11):2586-2595.

31. Peake JM, Della Gatta P, Suzuki K, et al.: Cytokine expression and secretion by skeletal muscle cells: Regulatory mechanisms and exercise effects. Exerc Immunol Rev 2015, 21:8-25.

32. Peeling P, Dawson B, Goodman C, et al.: Athletic induced iron deficiency: New insights into the role of inflammation, cytokines and hormones. Eur J Appl Physiol 2008, 103:381-91.

33. Peeling P, Sim M, Badenhorst CE, et al.: Iron status and the acute post-exercise hepcidin response in athletes. PLoS One 2014, 9:e93002.

34. Podgorski T, Krysciak J, Konarski J, et al.: Iron metabolism in field hockey players during an annual training cycle. J Hum Kinet 2015, 47:107-14.

35. Ponka P: Tissue-specific regulation of iron metabolism and heme synthesis: Distinct control mechanisms in erythroid cells. Blood 1997, 89:1-25.

36. Reif DW: Ferritin as a source of iron for oxidative damage. Free Radic Biol Med 1992, 12:417-27.

37. Robinson Y, Cristancho E, and Boning D: Intravascular hemolysis and mean red blood cell age in athletes. Med Sci Sports Exerc 2006, 38:480-3.

38. Rosa L, Cutone A, Lepanto MS, et al.: Lactoferrin: A natural glycoprotein involved in iron and inflammatory homeostasis. Int J Mol Sci 2017, 18.

39. Sandstrom G, Rodjer S, Jacobsson S, et al.: Increased level of serum hepcidin in female adolescent athletes. Clin J Sport Med 2018, 28:180-183.

40. Schoene RB, Escourrou P, Robertson HT, et al.: Iron repletion decreases maximal exercise lactate concentrations in female athletes with minimal iron-deficiency anemia. J Lab Clin Med 1983, 102:306-12.

41. Sim M, Garvican-Lewis LA, Cox GR, et al.: Iron considerations for the athlete: A narrative review. Eur J Appl Physiol 2019, 119:1463-1478.

42. Skarpanska-Stejnborn A, Basta P, Trzeciak J, et al.: Effect of intense physical exercise on hepcidin levels and selected parameters of iron metabolism in rowing athletes. Eur J Appl Physiol 2015, 115:345-51.

43. Soldin OP, Bierbower LH, Choi JJ, et al.: Serum iron, ferritin, transferrin, total iron binding capacity, hscrp, Idl cholesterol and magnesium in children; new reference intervals using the dade dimension clinical chemistry system. Clin Chim Acta 2004, 342:211-7.

44. Troadec MB, Laine F, Daniel V, et al.: Daily regulation of serum and urinary hepcidin is not influenced by submaximal cycling exercise in humans with normal iron metabolism. Eur J Appl Physiol 2009, 106:435-43.

45. Zugel M, Treff G, Steinacker JM, et al.: Increased hepcidin levels during a period of high training load do not alter iron status in male elite junior rowers. Front Physiol 2019, 10:1577. 
Figures
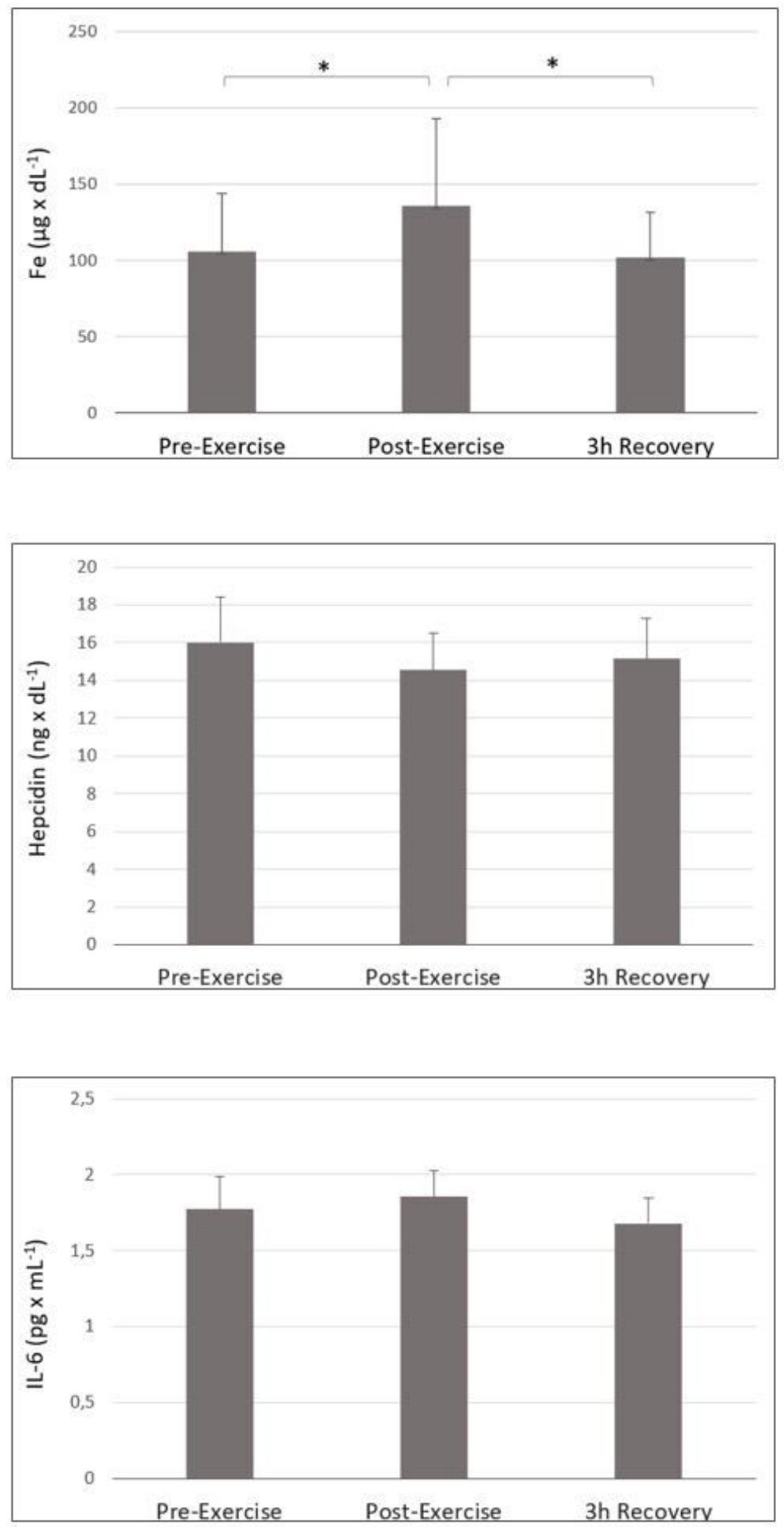

\section{Figure 1}

The effect of intense exercise on serum levels of iron, hepcidin, and IL-6 Note. Values are presented as the mean \pm SD. Significant difference at $* p<0.05$ 

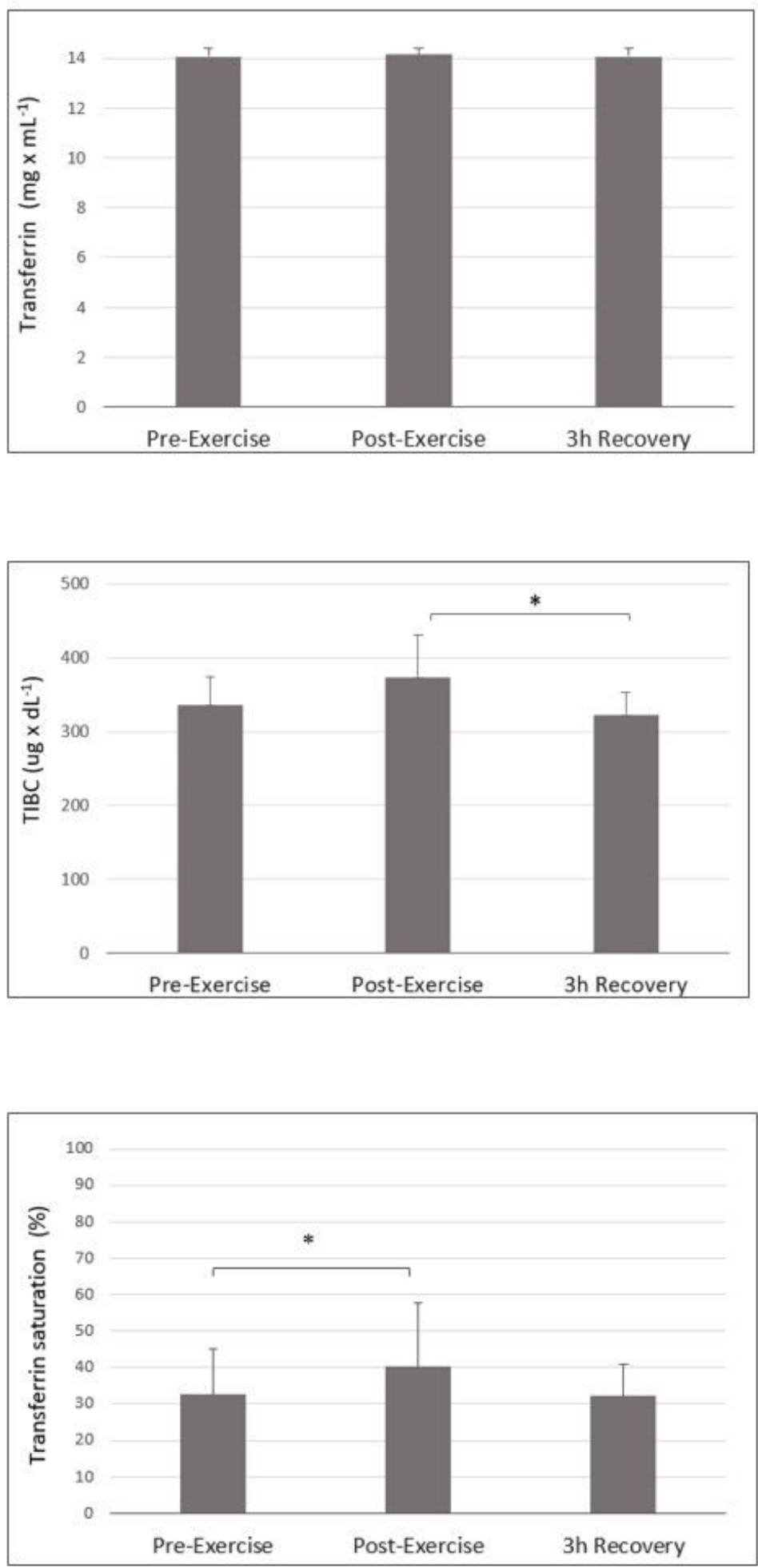

Figure 2

The effect of intense exercise on transferrin, TIBC, and transferrin saturation

Note. Values are presented as the mean \pm SD. Significant difference at ${ }^{\star} p<0.05$ 

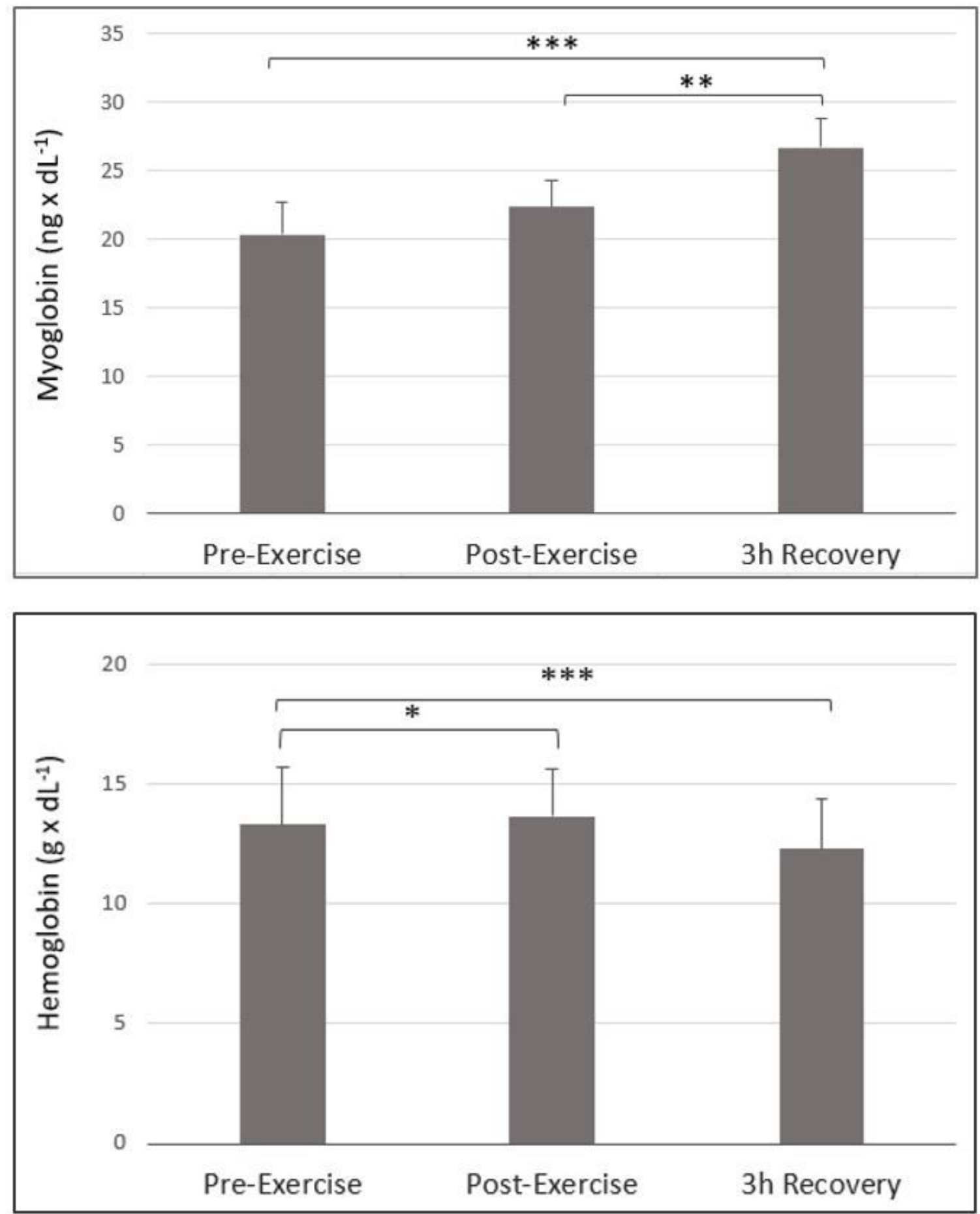

Figure 3

The effect of intense exercise on myoglobin and hemoglobin

Note. Values are presented as the mean \pm SD. Significant difference at ${ }^{\star} p<0.05$ and ${ }^{\star \star \star} p<0.001$ 

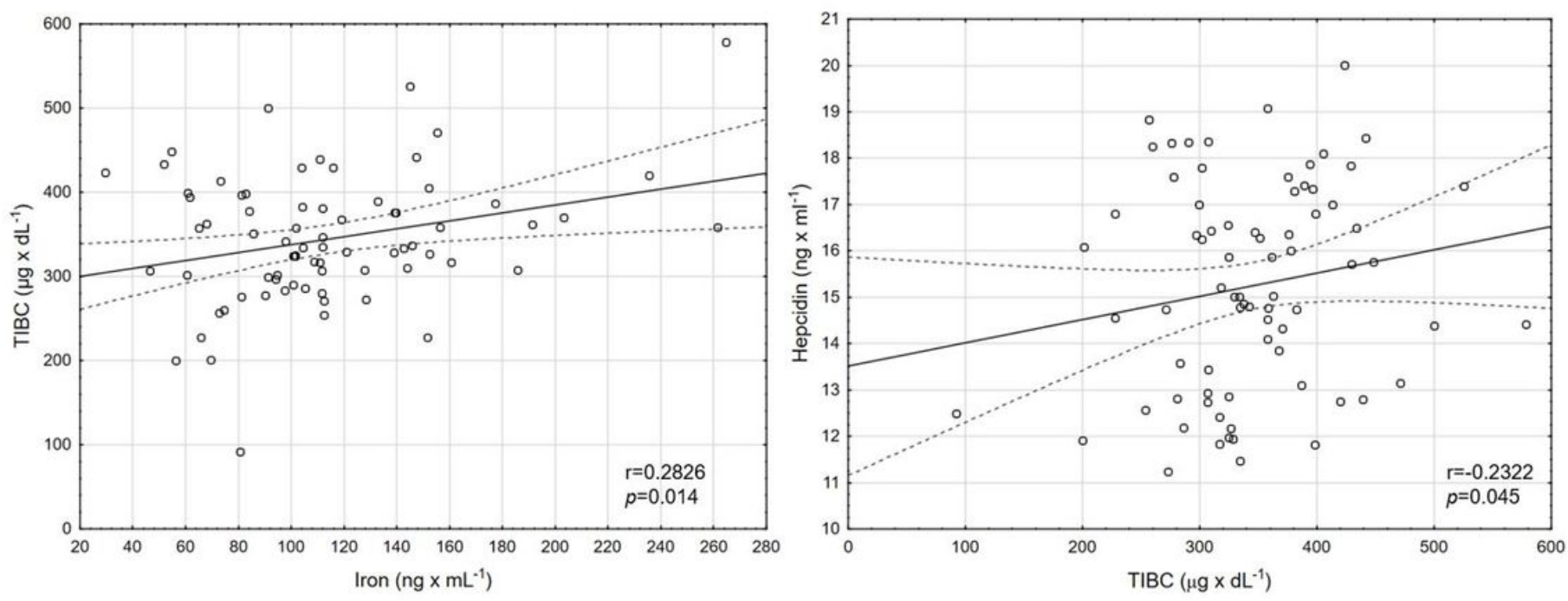

Figure 4

Correlation between iron and TIBC levels, and hepcidin and TIBC levels 